\title{
MATERIAL DISPERSION IN INTRINSIC GaAs FOR THE FAR-INFRARED RANGE
}

\author{
M. A. GRADO-CAFFARO* and M. GRADO-CAFFARO \\ Scientific Consultants, C/Julio Palacios, 11, 9-B, 28029-Madrid, Spain
}

(Received 25 July 1998; In final form 4 September 1998)

\begin{abstract}
An expression for the coefficient of material dispersion in intrinsic gallium arsenide for the far-infrared range is derived. From this result, the variation of the group index in the above range is discussed.
\end{abstract}

Keywords: Material dispersion; GaAs; far-infrared; group index

\section{INTRODUCTION}

Problems related to the variation of the refractive index of semiconductors in the infrared range play an important role since this phenomenon has interesting implications on charge-sensing optical probing systems [1-3]. In particular, let us consider intrinsic GaAs and GaAs integrated circuits to detect picosecond electric signals [3]. On the other hand, we recall that the change in the refractive index is associated with the well-known plasma-optical effect $[1-6]$; this effect is based upon the following fact: in a semiconductor the electrons of the conduction band and the holes of the valence band behave as free carriers.

The variation of the index of refraction gives rise to the existence of material dispersion. By virtue of this, the well-known concept of coefficient of material dispersion for optical fibers can be applied to photodetector devices as, for example, GaAs PIN photodiodes. This

*Corresponding author. 
coefficient for intrinsic GaAs submitted to far-infrared radiation will be the subject of the present paper. In particular, some associated aspects referring to group index will be discussed.

\section{VARIATION OF THE REFRACTIVE INDEX IN THE FAR-INFRARED RANGE}

Consider intrinsic GaAs in thermal equilibrium and Ref. [3], Eqs. (11) and (12), with $\alpha_{n} \approx \alpha_{p}(=\alpha)$ so that we have:

$$
\Delta \eta=\eta_{\infty}-\eta(\omega)=\frac{\eta_{\infty} \omega_{0}^{2}}{2 \omega^{2}}\left(1+\frac{\omega_{0}^{2}}{4 \omega^{2}}\right)
$$

with

$$
\omega_{0}^{2}=\frac{e^{2} n_{i} \alpha}{\varepsilon m^{*}}
$$

where $\eta(\omega)$ stands for index of refraction, $\omega$ is angular frequency, $\eta_{\infty}$ is the refractive index at very high frequencies $(\omega \rightarrow \infty), e$ is the electron charge, $\varepsilon$ is dielectric permittivity, $m^{*}$ is the reduced effective mass of an electron-hole pair, $n_{i}$ is the intrinsic carrier concentration, and $\alpha$ is a corrective factor corresponding to intrinsic carriers; this factor is introduced in order to take into account the dependence upon $n_{i}$ of $m^{*}$ (see Refs. [1-6]). On the other hand, from formula (2) we get the plasma resonant frequency namely:

$$
f_{0}=\frac{\omega_{0}}{2 \pi}=\frac{e}{2 \pi} \cdot\left(\frac{n_{i} \alpha}{\varepsilon m^{*}}\right)^{1 / 2}
$$

From Eq. (1) we get:

$$
\eta(\omega)=\eta_{\infty}\left[1-\frac{\omega_{0}^{2}}{2 \omega^{2}}\left(1+\frac{\omega_{0}^{2}}{4 \omega^{2}}\right)\right]
$$

and by virtue of $\omega=2 \pi c / \lambda$ ( $c$ and $\lambda$ denote speed of light in vacuum and wavelength, respectively), from Eq. (4) it follows:

$$
\eta(\lambda)=[\eta]_{\lambda=0} \cdot\left[1-\frac{\omega_{0}^{2} \lambda^{2}}{8 \pi^{2} c^{2}}\left(1+\frac{\omega_{0}^{2} \lambda^{2}}{16 \pi^{2} c^{2}}\right)\right]
$$


so that we find:

$$
\frac{d}{d \lambda}(\eta(\lambda))=-\frac{[\eta]_{\lambda=0} \cdot \omega_{0}^{2} \lambda}{4 \pi^{2} c^{2}}\left(1+\frac{\omega_{0}^{2} \lambda^{2}}{8 \pi^{2} c^{2}}\right)
$$

Notice that $(d \eta / d \lambda)<0$, that is, dispersion is normal. Expression (6) is approximately valid for the far-infrared range (see Ref. [3]).

\section{MATERIAL DISPERSION}

Let us consider the well-known expression for the coefficient of material dispersion namely:

$$
M(\lambda)=\frac{\lambda}{c} \cdot \frac{d^{2}}{d \lambda^{2}}(\eta(\lambda))
$$

so that by Eqs. (6) and (7) it follows:

$$
M(\lambda)=-\frac{\omega_{0}^{2} \cdot[\eta]_{\lambda=0} \cdot \lambda}{4 \pi^{2} c^{3}}\left(1+\frac{3 \omega_{0}^{2} \lambda^{2}}{4 \pi^{2} c^{2}}\right)
$$

From the parabolic relationship (8) it is easy to see that there are no finite and positive wavelengths (that is, wavelengths with physical meaning) such that $M=0$ at these wavelengths. In contrast, for fused silica optical fibers, the coefficient of material dispersion is null for $\lambda \approx 1.3 \mu \mathrm{m}$. These facts show the discrepancies, with respect to material dispersion, between optical fibers and GaAs PIN photodiodes. On the other hand, note the negativeness of $M$ in Eq. (8).

Next we shall examine the group index, $\eta_{g}$, for intrinsic GaAs. We have:

$$
\eta_{g}=\eta-\lambda \frac{d \eta}{d \lambda}
$$

so that by Eqs. (6) and (9) it follows:

$$
\eta_{g}(\lambda)=[\eta]_{\lambda=0} \cdot\left[1+\frac{\omega_{0}^{2} \lambda^{2}}{8 \pi^{2} c^{2}}\left(1+\frac{3 \omega_{0}^{2} \lambda^{2}}{16 \pi^{2} c^{2}}\right)\right]
$$


In addition, by Eqs. (7) and (9) it is derived:

$$
M(\lambda)=-\frac{1}{c} \cdot \frac{d \eta_{g}}{d \lambda}
$$

Since $M(\lambda)<0$, by (11) it follows that $\left(d \eta_{g} / d \lambda\right)>0$ as expected from direct differentiation in formula (10); thus the group index increases as wavelength increases. Next we will define the relative group-index perturbation namely $\Delta \eta_{g} / \eta_{g}(0)=\left(\eta_{g}(\lambda)-\eta_{g}(0)\right) / \eta_{g}(0)$ so that by Eqs. (9) and (10) we get:

$$
\frac{\Delta \eta_{g}(\lambda)}{\eta_{g}(0)}=\frac{\omega_{0}^{2} \lambda^{2}}{8 \pi^{2} c^{2}}\left(1+\frac{3 \omega_{0}^{2} \lambda^{2}}{16 \pi^{2} c^{2}}\right)
$$

From expression (12) it is easy to infer that the above perturbation increases as frequency decreases.

\section{CONCLUSIONS}

The preceding analysis is useful to evaluate certain aspects of the optical behaviour of intrinsic GaAs for the far-infrared range. In fact, an expression for the material dispersion coefficient has been obtained. Moreover, group index has been examined; in particular, a relationship for the relative group-index perturbation has been found. This perturbation constitutes a useful parameter to estimate the performance of charge-sensing optical probing systems. Finally, we want to remark that in our preceding calculations the plasma resonant frequency plays an important role; for intrinsic GaAs at room temperature and neglecting the concentration-dependence of effective mass, we have obtained $f_{0} \approx 13.49 \mathrm{MHz}$ by using Eq. (3) (we have taken $\alpha=1, n_{i} \approx 1.8 \times 10^{12} \mathrm{~m}^{-3}$ ).

\section{References}

[1] Keller, U., Diamond, S. K., Auld, B. A. and Bloom, D. M. (1987). Appl. Phys. Lett., 53, 388.

[2] Heinrich, H. K. (1990). IBM J. Res. Dev., 34(2/3), 164.

[3] Grado-Caffaro, M. A. and Grado-Caffaro, M. (1993). Act. Pass. Electronic Comp., $15,63-66$. 
[4] Wooten, F. (1972). “ Optical properties of solids" (Academic Press, New York, pp. 52-55).

[5] Soref, R. A. and Bennett, B. R. (1987). IEEE J. Quantum Electron., QE-23, 123.

[6] Koskowich, G. N., Darling, R. B. and Soma, M. (1988). Phys. Rev., B38, 1281. 

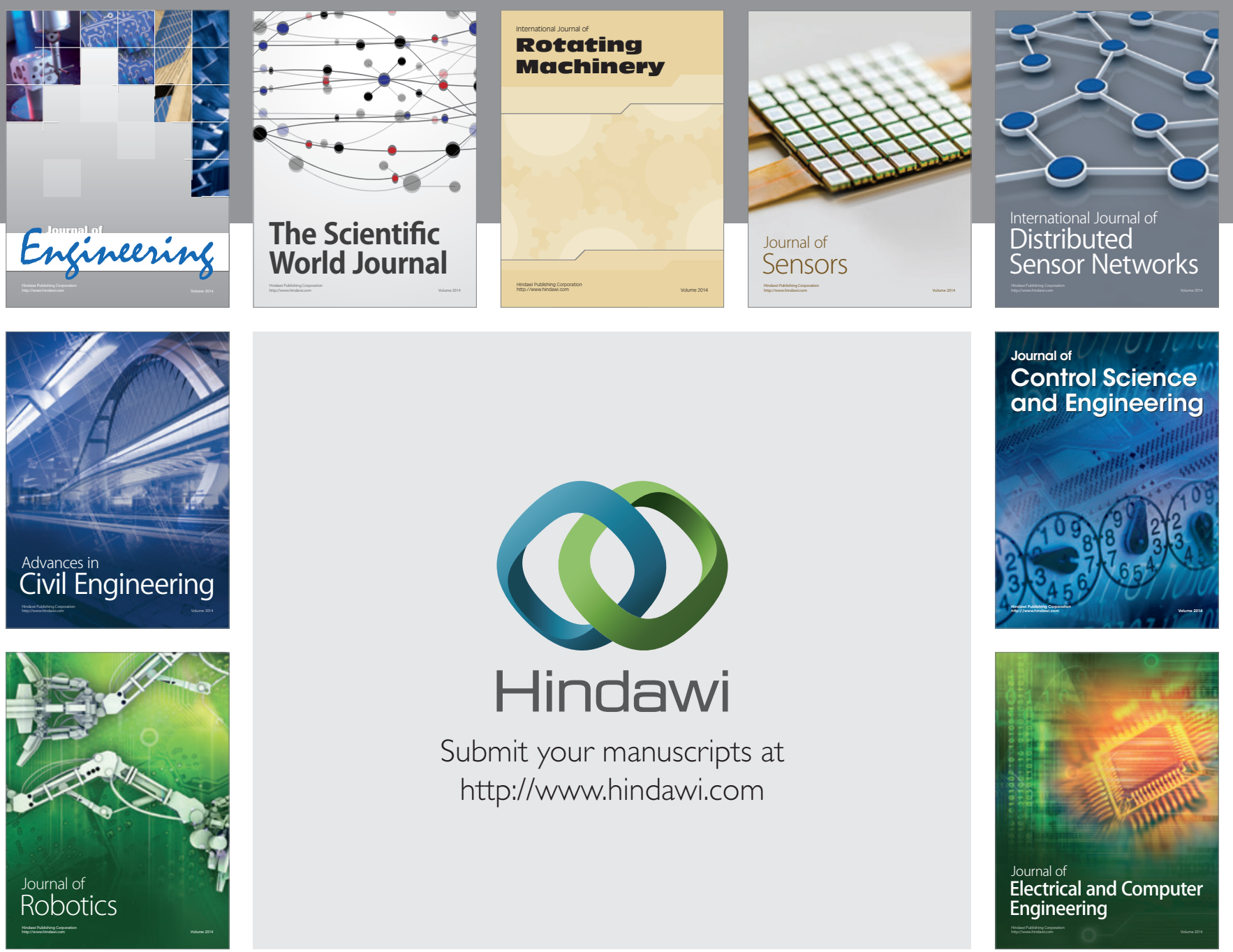

Submit your manuscripts at

http://www.hindawi.com
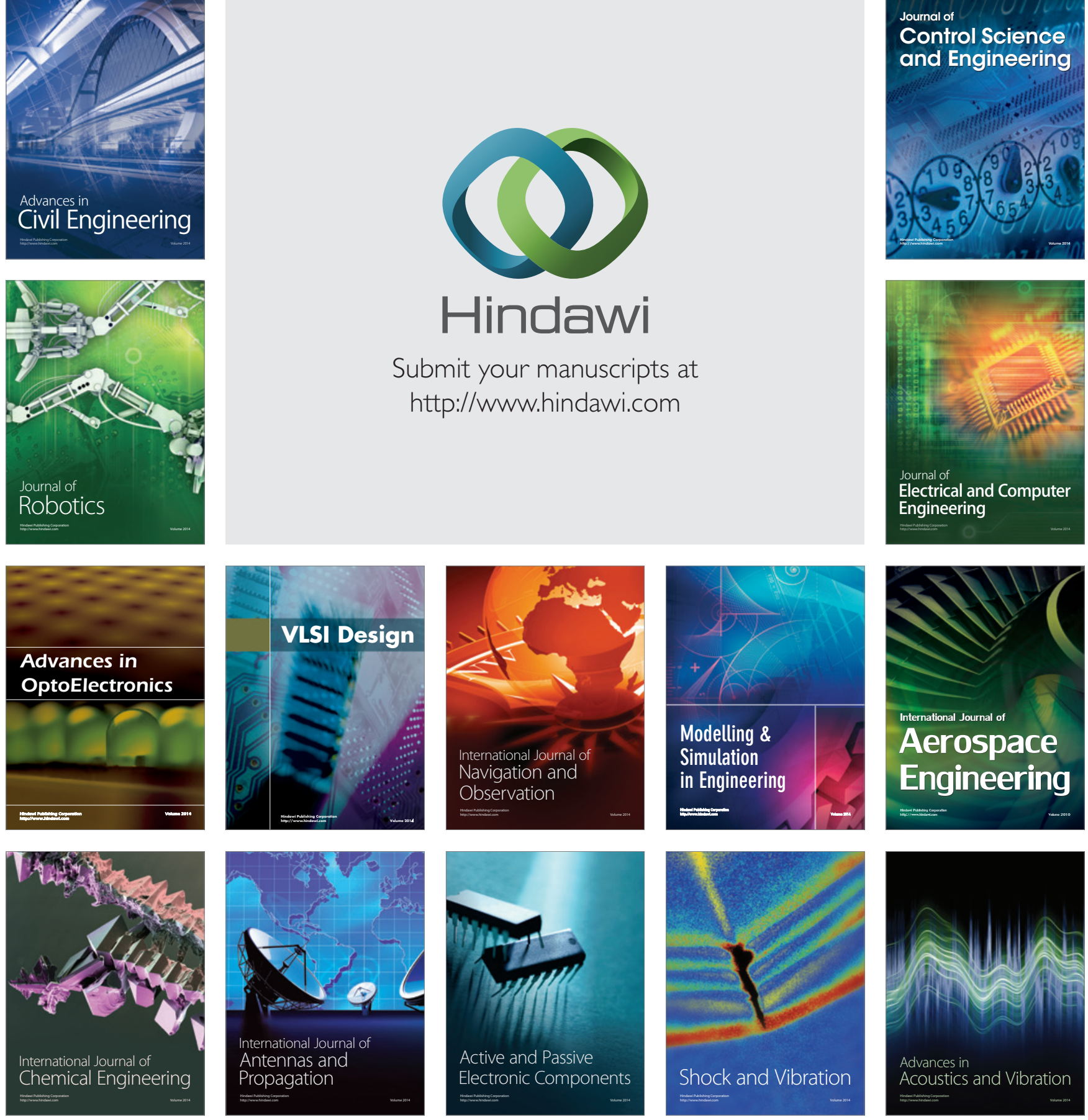